\title{
Change of precipitation intensity spectra at different spatial scales under warming conditions
}

\author{
WU FuTing ${ }^{1} \&$ FU CongBin ${ }^{1,2 *}$ \\ ${ }^{1}$ Institute for Climate and Global Change Research, School of Atmospheric Sciences, Nanjing University, Nanjing 210093, China; \\ ${ }^{2}$ START/TEA, Institute of Atmospheric Physics, Chinese Academy of Sciences, Beijing 100029, China
}

Received June 25, 2012; accepted October 8, 2012; published online February 27, 2013

\begin{abstract}
The long-term change of the whole spectra of precipitation intensity in China is examined using observed daily data recorded at 477 surface stations for the period from 1961 to 2008. The results show a spatially coherent decrease of trace precipitation despite different reduction magnitudes among the regions. For measurable precipitation, significant regional and seasonal characteristics are observed. In autumn, the whole measurable precipitation decreased over Eastern China (east of $98^{\circ} \mathrm{E}$ ). In summer and winter, a significant increase of heavy precipitation and decrease of light precipitation are detected south of Eastern China. In Western China, measurable precipitation is found to have increased in all four seasons. Composite analysis reveals a quasi-linear relationship between increasing surface temperature and precipitation on a global scale. The responses of precipitation at different intensities to the increased temperature are distinct, with a significant spectra-shifting from light to heavy precipitation. Compared with precipitation over the ocean, the amplification of heavy precipitation over land is relatively less, most likely constrained by the limited water supply. The response of regional precipitation to global warming shows greater uncertainties compared with those on the global scale, perhaps due to interference by more complex topography and land cover, as well as human activities, among other factors.
\end{abstract}

precipitation spectra, global warming, spatial scale, extreme precipitation, composite analysis

Citation: Wu F T, Fu C B. Change of precipitation intensity spectra at different spatial scales under warming conditions. Chin Sci Bull, 2013, 58: 1385-1394, doi: $10.1007 / \mathrm{s} 11434-013-5699-0$

In practice, precipitation changes at different intensities are of substantial interest to human society because their impacts are distinct. Consecutive and steady light and moderate precipitation can effectively soak into the soil and alleviate drought, while heavy precipitation in a short period, such as torrential rain, may cause flooding and soil erosion [1]. The formation mechanisms of these precipitation types are also different. Heavy precipitation usually forms in fastgrowing convective clouds, which are intimately connected with robust moisture rising caused by dynamic convergence, orographic uplifting or surface heating [2-4]. In contrast, low-intensity precipitation is more associated with cloud microphysical processes, which are affected by aerosols, moisture content and so on [5-8].

\footnotetext{
*Corresponding author (email: fcb@nju.edu.cn)
}

The ever-increasing global mean surface temperature has been widely recognized as an important factor in increased extremely heavy precipitation [1,9]. On a regional scale, many studies have also attributed the frequently occurring flood disasters to global warming $[4,10,11]$. However, there is still no consensus in terms of quantitative estimates regarding the contribution by increased temperature. A theoretically well-determined value is the change in waterholding capacity of the atmosphere of $7 \% / \mathrm{K}$, governed by the Clausius-Clapeyron equation [1]. This equation indicates an increase of precipitable water (column-integrated water) in relation to the constant relative humidity on the global scale [12]. This value is at odds with the model-predicted increase of $2 \% / \mathrm{K}$ in precipitation intensity proposed in a study using an ensemble of 17 latest-generation climate models [13]. Locally, Lenderink and Meijgaard [14], using 
hourly precipitation observations from De Bilt in the Netherlands, found that precipitation extremes increased with rising temperatures twice as fast as expected theoretically from the Clausious-Clapeyron equation when the daily mean temperature exceeds $12^{\circ} \mathrm{C}$. This result can be explained by Trenberth et al. [1], who indicated that additional latent heat released from precipitation invigorated the precipitation system and further enhanced the convergence of moisture.

As shown above, there have been many studies on extreme precipitation, especially for very heavy precipitation events $[1,10,15,16]$. Meanwhile, studies on light precipitation over China also have made substantial achievements and have found a significant decrease of light precipitation in the last several decades $[17,18]$. Some studies have attributed the decrease of light precipitation to increased temperature [17,19], while others have argued that the increased aerosol pollution has led to the reduction of light precipitation through cloud microphysical processes [8]. However, more detailed analyses need to be made regarding changes and their possible explanations in the whole precipitation spectra, ranging from light to extremely heavy, on different spatial scales.

In this study, we first examine the changes of the whole precipitation spectra in China based on observed daily precipitation during the period from 1961 to 2008. In the second part, we investigate the variation features of precipitation amounts of 10 categories at both regional and global scales in a warming scenario and compare their connections with increasing global mean surface temperature using a recently developed analysis method by Liu et al. [20], which has been shown to be able to detect quantitatively the relationship between increasing temperature and the variation of precipitation.

\section{Data and methods}

\subsection{Data}

Daily precipitation data at 753 surface stations from 1961 to 2009 are provided by the National Meteorological Information Centre of the China Meteorological Administration (NMIC/CMA) and have been strictly quality controlled. For the period from 1961 to 2009, some stations have missing data records. Considering that consistency and completeness of the data are very important for the trend calculation, we excluded 276 stations with missing records during the study period, leaving 477 stations for the present study. Figure 1 shows the locations of these stations. In view of the regional differences of precipitation, we divided the 477 stations into eight subregions, first according to the longitude and latitude (Table 1), which to some degree are consistent with climate regimes: Northeast (NE), North China (NC), the middle and lower reaches of the Yangtze River (YZ), Southeast (SE), the upper reaches of the Yellow River

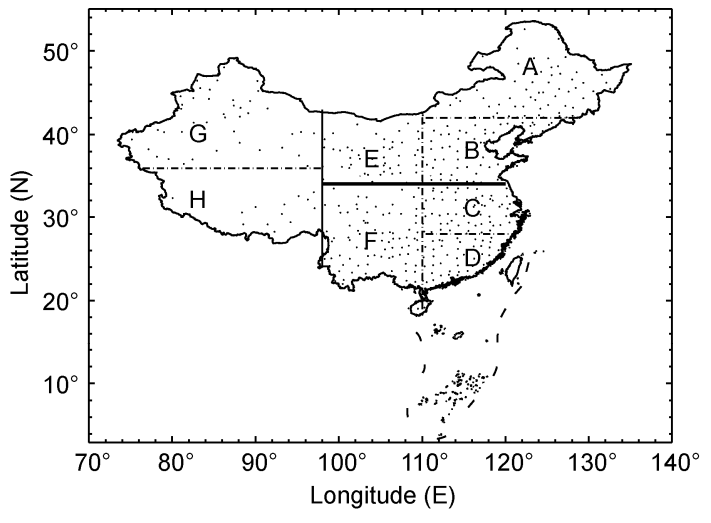

Figure 1 Station distribution in China and eight subregions. Subregions: A, northeast (NE); B, north China (NC); C, lower reaches of the Yangtze River (YZ); D, southeast (SE); E, upper reaches of the Yellow River (YW); F, southwest (SW); G, northwest (NW); H, east of Tibet (TB). The solid lines divide the map into three major areas: Area I (NE, NC and YW subregions), Area II (YZ, SE, and SW subregions), and Area III (NW and TB subregions).

Table 1 Eight subregions divided by longitude and latitude

\begin{tabular}{lcc}
\hline \multicolumn{1}{c}{ Region } & Longitude $\left({ }^{\circ} \mathrm{E}\right)$ & Latitude $\left({ }^{\circ} \mathrm{N}\right)$ \\
\hline Northeast & $\geqslant 110$ & $>42$ \\
North China & $\geqslant 110$ & {$[34,42]$} \\
Middle and lower reach of the & $\geqslant 110$ & {$[28,34]$} \\
$\quad$ Yangtze River & $\geqslant 110$ & $<28$ \\
Southeast & {$[98,110]$} & $>34$ \\
Upper reach of the Yellow River & {$[98,110]$} & $\leqslant 34$ \\
Southwest & $<98$ & $\leqslant 36$ \\
Northwest & $<98$ & $<36$ \\
East of Tibet &
\end{tabular}

(YW), Southwest (SW), Northwest (NW) and east of Tibet (TB). Precipitation is measured at the stations twice daily at 0000 and 1200 UTC (0800 and 2000 Beijing Time). The Chinese standard precipitation gauge (CSPG) has been the standard instrument for measuring both solid and liquid precipitation in China's climatological and hydrological stations networks since the 1950s [18]. A precipitation event of $<0.1 \mathrm{~mm}$ is beyond the resolution of the CSPG and is recorded as a trace amount of precipitation [21]. In this study, we considered all precipitation events, including measurable precipitation $(>0.1 \mathrm{~mm})$ and trace precipitation events.

In addition, blended space-based and ground-based global rainfall datasets from the Global Precipitation Climatology Project (GPCP) during the period from 1979 to 2007 were employed to study the change of precipitation spectra on a global scale and to investigate the relationship between precipitation change and global warming. The spatial resolution of the GPCP is $2.5^{\circ} \times 2.5^{\circ}$, and the temporal resolution is total precipitation in five consecutive days (pentad) [22]. Global temperatures are provided by the National Oceanic and Atmospheric Administration/National Climatic Data 
Center (NOAA/NCDC), produced from blended land and ocean datasets [23]. This dataset provides temperature anomalies from 1880 to the present, with respect to the period from 1901 to 2000 (i.e. the 20th century average). Here, we only apply the data during the study period.

\subsection{Methods}

A linear fitting method is used to estimate the trend of time series for each precipitation category. It should be noted that this definition does not necessarily imply a "real trend" in the sense of a long-term climate change time scale. More accurately, the trend only presents a monotonic shift during the period studied. In analyzing the implications of global warming in precipitation spectra, we follow a relatively new technique that uses the differences of the precipitation amount of each category and global temperatures between every two years instead of pure series analysis. According to the difference of temperature, matches of each precipitation bin in the same two years are sorted. Our analysis is only based on the series of precipitation matches. In this way, quantitative contributions of global temperatures to precipitation can be extracted despite various factors that may affect precipitation characteristics. This method was first introduced by Liu et al. [20].

To compare the similarities and differences of the implications between different spatial scales, we categorized 10 bins of precipitation intensity according to the equal contribution to the total accumulated precipitation amount over a specific area (e.g. Fujibe et al. [10]). The categories vary depending on the region and chosen period. Table 2 lists the boundaries of each bin over $60^{\circ} \mathrm{S}-60^{\circ} \mathrm{N}$ and $20^{\circ}-40^{\circ} \mathrm{N}$ as well as combined land-and-ocean areas within $20^{\circ}-40^{\circ} \mathrm{N}$. It can be observed that the upper limits over ocean areas are larger than those over land for low-intensity bins (Bin 1Bin 5); however, they are smaller for medium and heavy precipitation bins (Bin 6-Bin 9). The same characteristic is found when comparing the intensity range over $20^{\circ}-40^{\circ} \mathrm{N}$ with that over $60^{\circ} \mathrm{S}-60^{\circ} \mathrm{N}$, which implies that light precipitation over land contributes more than that over ocean. On the other hand, heavy precipitation is less strong over land than over ocean, so a wider range of precipitation is needed to make the same contribution (in terms of amount), which is consistent with less water vapor supply over land.

\section{Results and analysis}

\subsection{Precipitation spectral change in China}

Impacted by a typical monsoon climate, the precipitation in China shows significant regional and seasonal characteristics. From southeast to northwest, the precipitation amount generally exhibits a decreasing tendency on the whole. Seasonally, precipitation exhibits a peak in summer due to the summer monsoon (Figure 2). Furthermore, the ranges of precipitation intensity are also distinct over different subregions in the four seasons. Figure 2 compares the distribution of precipitation spectra over the southeast and northwest of China, averaged during the period from 1961 to 2008. Obviously, the range of precipitation spectra is much wider in the southeast of China than that over the northwest, with the intensity extending from 0.1 to $200 \mathrm{~mm}$ and even more. By contrast, the precipitation over West China consists mainly of light and moderate intensities ranging from 0.1 to $30 \mathrm{~mm}$; more intense precipitation is rather rare.

Considering the different ranges of precipitation intensity, we define the precipitation intensity maximum boundary according to the precipitation spectral distribution (Figure 2) over the region seasonally to ensure the analysis of the trend is effective. Examination shows that changes of precipitation spectra can be grouped into three major areas: (I) north of Eastern China, consisting of the NE, NC and YW subregions; (II) south of Eastern China, consisting of the YZ, SE, and SW subregions; (III) West China, including the NW and TB subregions. The three major regions are shown in Figure 1 with bold solid lines.

According to the Chinese national meteorological standards, the measurable daily rain rates are classified into five grades of intensity: small $(0.1-10 \mathrm{~mm} / \mathrm{d})$, medium (10-25 $\mathrm{mm} / \mathrm{d})$, large $(25-50 \mathrm{~mm} / \mathrm{d})$, heavy $(50-100 \mathrm{~mm} / \mathrm{d})$, and very heavy $(>100 \mathrm{~mm} / \mathrm{d})$ rains. Considering the regional discrepancies of precipitation, the defined maximum boundary varies over different subregions in the four seasons in our study. Specific boundaries for the eight subregions (three major areas) are shown in Table 3. Given that small precipitation accounts for most of the total precipitation, it is further categorized into 10 grades in $1 \mathrm{~mm}$ increments. We calculated the frequency of trace precipitation and the amount of each grade of measurable precipitation and examined the long-term trend from 1961 to 2008 over

Table 2 Boundaries of 10 categories over $60^{\circ} \mathrm{S}-60^{\circ} \mathrm{N}, 20^{\circ}-40^{\circ} \mathrm{N}$, together with the ocean and land areas in the mid latitude over the Northern Hemisphere $(\mathrm{mm} / \mathrm{d})$

\begin{tabular}{lccccccccccc}
\hline & Bin 1 & Bin 2 & Bin 3 & Bin 4 & Bin 5 & Bin 6 & Bin 7 & Bin 8 & Bin 9 & Bin 10 \\
\hline $60^{\circ} \mathrm{S}-60^{\circ} \mathrm{N}$ & $<1.68$ & 2.78 & 3.82 & 4.94 & 6.23 & 7.78 & 9.79 & 12.6 & 17.6 & $\geqslant 17.6$ \\
$20^{\circ}-40^{\circ} \mathrm{N}$ (ocean) & $<1.53$ & 2.73 & 3.89 & 5.11 & 6.42 & 7.92 & 9.78 & 12.4 & 17.1 & $\geqslant 17.1$ \\
$20^{\circ}-40^{\circ} \mathrm{N}$ & $<1.46$ & 2.65 & 3.82 & 5.04 & 6.38 & 7.92 & 9.86 & 12.6 & 17.4 & $\geqslant 17.4$ \\
$20^{\circ}-40^{\circ} \mathrm{N}$ (land) & $<1.30$ & 2.43 & 3.59 & 4.85 & 6.27 & 7.95 & 10.1 & 13.1 & 18.3 & $\geqslant 18.3$ \\
\hline
\end{tabular}



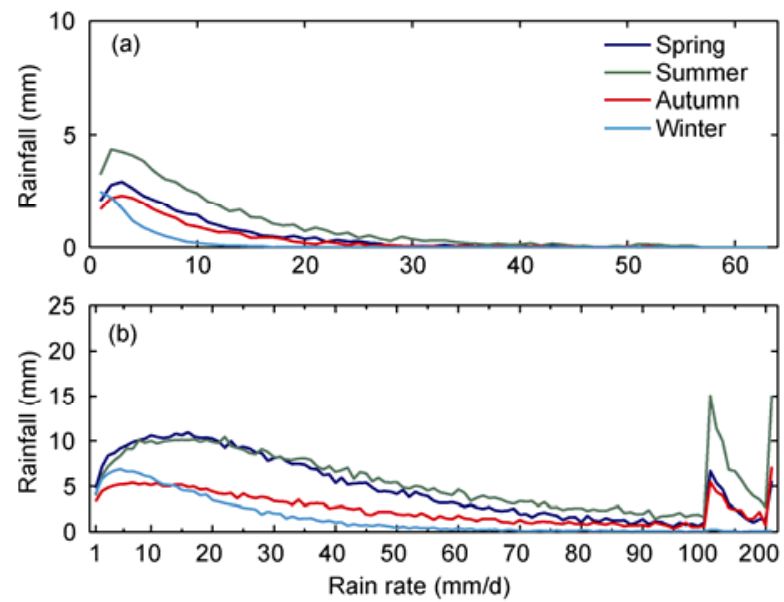

Figure 2 Distribution of precipitation intensity spectra over Northwest China (a) and the Southeast China (b) for different seasons.

Table 3 Maximum boundaries of precipitation intensity over eight different regions in four seasons $(\mathrm{mm} / \mathrm{d})$

\begin{tabular}{lccc}
\hline & Area I & Area II & Area III \\
\hline Spring & $\geqslant 50$ & $\geqslant 100$ & $\geqslant 25$ \\
Summer & $\geqslant 100$ & $\geqslant 100$ & $\geqslant 50$ \\
Autumn & $\geqslant 50$ & $\geqslant 100$ & $\geqslant 25$ \\
Winter & $\geqslant 10$ & $\geqslant 50$ & $\geqslant 10$ \\
\hline
\end{tabular}

the eight subregions.

(i) Trace precipitation. Trace precipitation (also called drizzle) cannot be detected by a rain gauge but can be perceived by the human body. Trace precipitation reflects the dry/wet condition of atmosphere to some degree [24] and plays an important role in the replenishment of soil. Figure 3 demonstrates the long-term trend of trace precipitation frequency over the eight subregions in different seasons during the period from 1961 to 2008 . The trend analysis of trace precipitation frequency from 1961 to 2008 shows a consistent and uniform decreasing trend over China in all

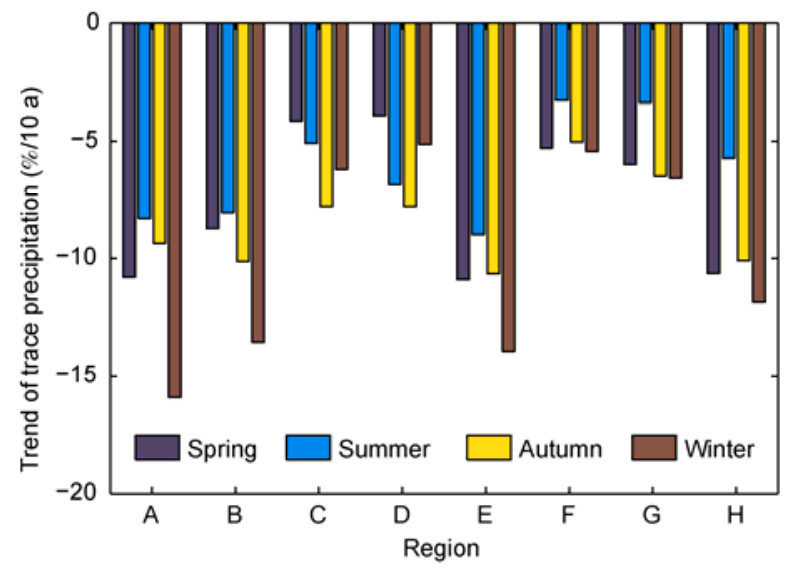

Figure 3 Trend of trace precipitation frequency over eight subregions in China for four seasons. four seasons. In fact, several studies have drawn the same conclusion [17,19,24,25]. Liu et al. [18] further indicated that trace precipitation events decreased abruptly starting in 1982 when the temperature started rising rapidly in China. In this study, we find that, quantitatively, the trace precipitation over NC, NE, YW and TB has decreased by approximately $10 \% / 10 \mathrm{a}$, which is twice the rate of decrease in South China $(-5 \% / 10$ a, where the negative sign indicates reduction), including YZ, SE and SW. Seasonally, the trace precipitation frequency decreased most strongly in winter, a trend perhaps connected with the different increasing rates of local temperatures.

(ii) Measurable precipitation. Figure 4 presents the trend of precipitation spectra over Area I (consisting of subregions $\mathrm{NE}, \mathrm{NC}$ and $\mathrm{YW}$ ) in four different seasons from 1961 to 2008. It can be observed that the precipitation spectral trend exhibits significant seasonal and regional characteristics. In spring, almost all of the precipitation spectra show an increasing trend over NE, especially for the small intensity category. Over the NC and YW subregions, large and heavy precipitation has increased, while small and moderate precipitation shows a decreasing trend. In summer and autumn, all types of precipitation exhibit a uniform decreasing trend except for the moderate precipitation (8-25 $\mathrm{mm} / \mathrm{d}$ ) and very heavy precipitation categories over the YW subregion in summer. Wang and Yan [26] analyzed the trends of seasonal precipitation over China from 1951 to 2007 and found a changing pattern of extreme precipitation consistent with the present study. They also noted that the roles of large-scale and regional mechanisms are quite different for different seasons. A significant decrease of precipitation in summer related to the weakening of the summer monsoon can explain the persistent drought in North China during recent years. As a typical region dominated by the well-known East Asian monsoon, a prominent interdecadal shift in the East China rainfall pattern occurred in late 1970 with more precipitation in the Yangtze River Valley and less in North China, which has been attributed to the weakening of the Asian summer monsoon [27]. Li et al. [28] also investigated the extreme precipitation and ascribed the greater precipitation extremes in the northern part of eastern China to variations of the East Asian summer monsoon and corresponding atmospheric circulations. In winter, the daily precipitation amount that exceeds $10 \mathrm{~mm}$ is uncommon over this subregion. However, our trend analysis shows a consistent and considerable increase of widespread precipitation in winter, which is obviously different from that in summer and autumn.

Figure 5 shows the trend of precipitation spectra over Area II (including YZ, SE and SW) from 1961 to 2008. This area has a typical monsoon climate. During warm months (May-October), strong moisture transported from the ocean is the main precipitation source over this area. As shown in Figure 5, a significant feature over this area is the uniform reduction of the whole precipitation spectra in 

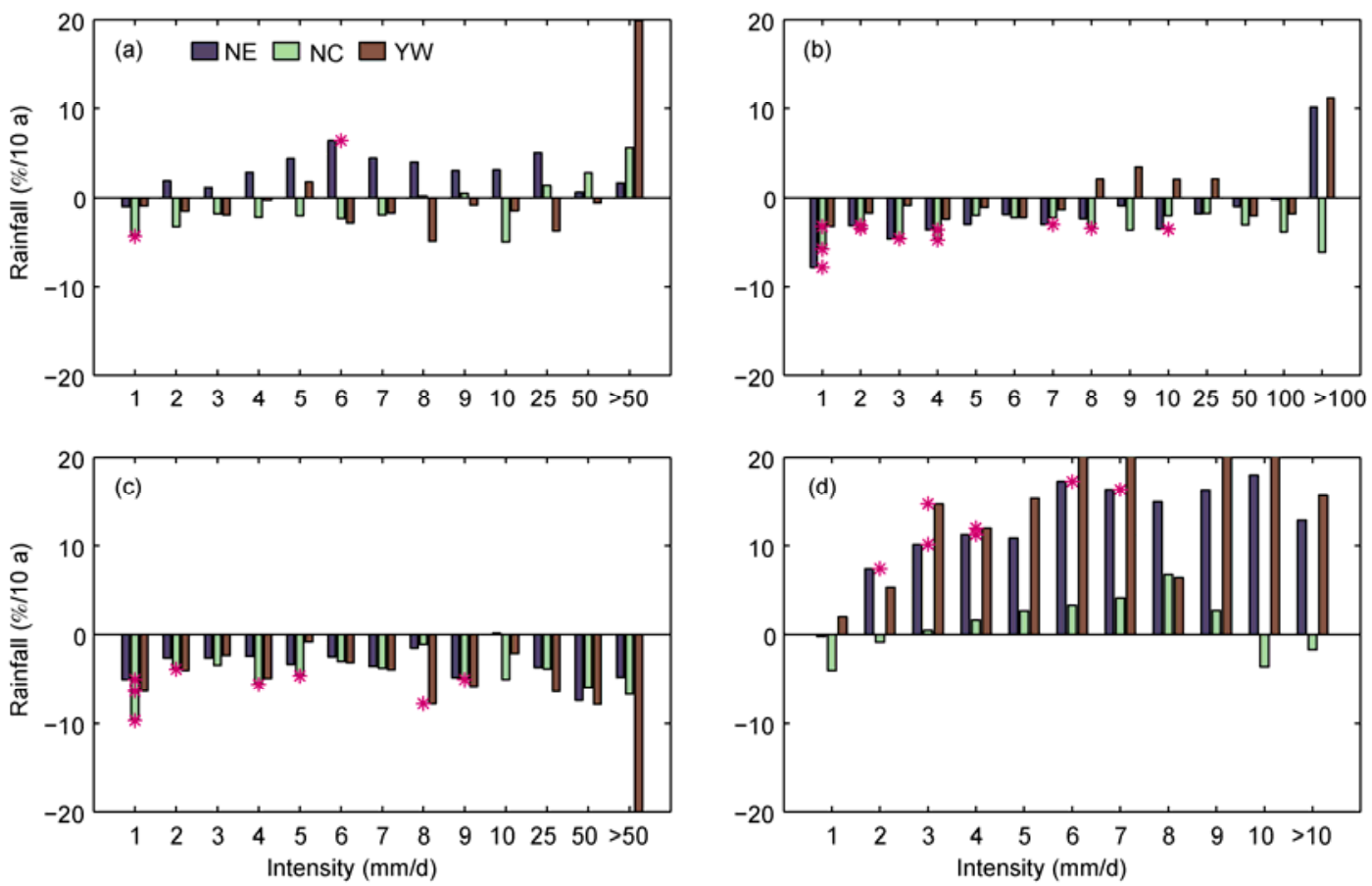

Figure 4 Trend of precipitation for different intensity categories over north of eastern China (Area I, including northeast, north China and upper reaches of the Yellow River) during 1961-2008. (a) Spring; (b) summer; (c) autumn; (d) winter. An asterisk (*) indicates that the trend is statistically significant at the $95 \%$ confidence level.
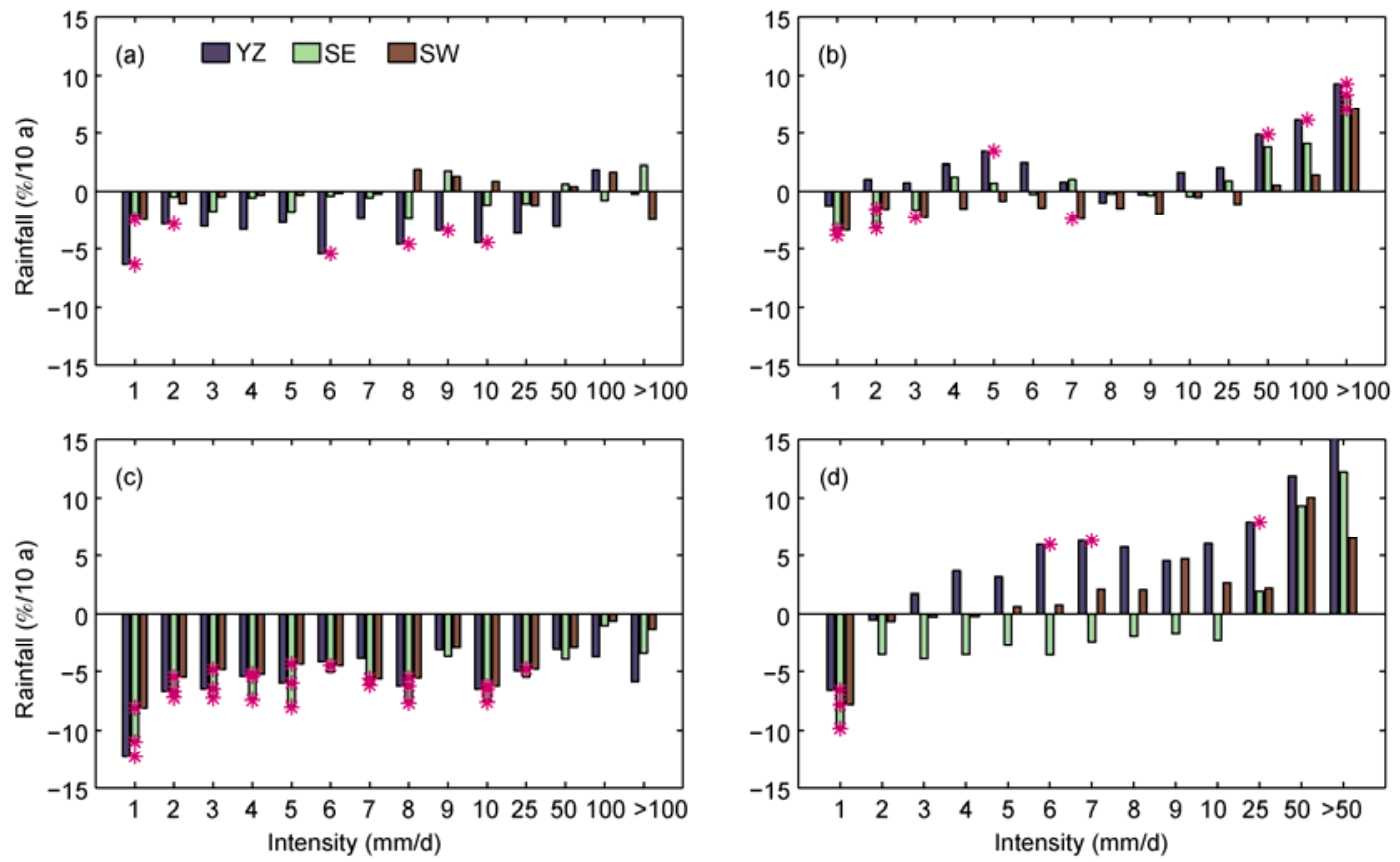

Figure 5 Same as in Figure 4, except for south of eastern China (Area II), including the lower reaches of the Yangtze River, southeast China, and southwest China.

autumn, which is consistent with that over Area I. The decreasing magnitude of the small precipitation is greater than that of the heavy precipitation. In spring, the daily precipitation less than $7 \mathrm{~mm}$ shows a uniform decreasing trend over the three subregions, with the maximum reduction in YZ. For other categories, the precipitation in spring shows dis- tinct characteristics over the three subregions, although the trend is not significant. In summer, the significant increase in heavy precipitation and decrease in small, especially light precipitation over SE and SW can be found. The increasing magnitude of large, heavy and very heavy precipitation is the largest in YZ, exceeding 5\%/10 a. Furthermore, the 
increasing magnitude is also connected with the precipitation intensity. Heavier precipitation tends to have a greater increase, the so-called "the richer gets richer" effect. It should be noted that there is also some difference even within a precipitation intensity less than $10 \mathrm{~mm} / \mathrm{d}$ in summer. An increasing trend is detected for precipitation between 4 and $7 \mathrm{~mm} / \mathrm{d}$ over YZ and SE. Similar results were reported by Liu et al. [18]. They mainly attributed the decline in precipitation to the decrease of light precipitation events with intensities of $0.1-0.3 \mathrm{~mm} / \mathrm{d}$. In winter, the whole precipitation spectra show a significant increasing trend. Only in SE, with precipitation less than $2 \mathrm{~mm} /$ day and more than $9 \mathrm{~mm} / \mathrm{d}$, has the precipitation declined during 1961-2008.

In West China (Area III), the total precipitation is rather limited, resulting in an arid and semiarid climate. Recent studies have found an increasing trend of total precipitation in this area [29]. Figure 6 shows the trend of precipitation spectra in area III, including the northwest (NW) and Tibetan region (TB). One can see that nearly all intensities of precipitation have increased in all four seasons, which confirms the wet tendency in West China reported in the literature [29-32]. Li et al. [31] argued that the direct cause of the shift from a warm-dry to warm-wet climate is the adjustment of atmospheric circulation, which strengthens the water-vapor transport to the north from the Indian Ocean and the western Pacific Ocean.

\subsection{Global precipitation spectral change}

To compare precipitation features at different spatial scales, we also examine the change of precipitation spectra on the
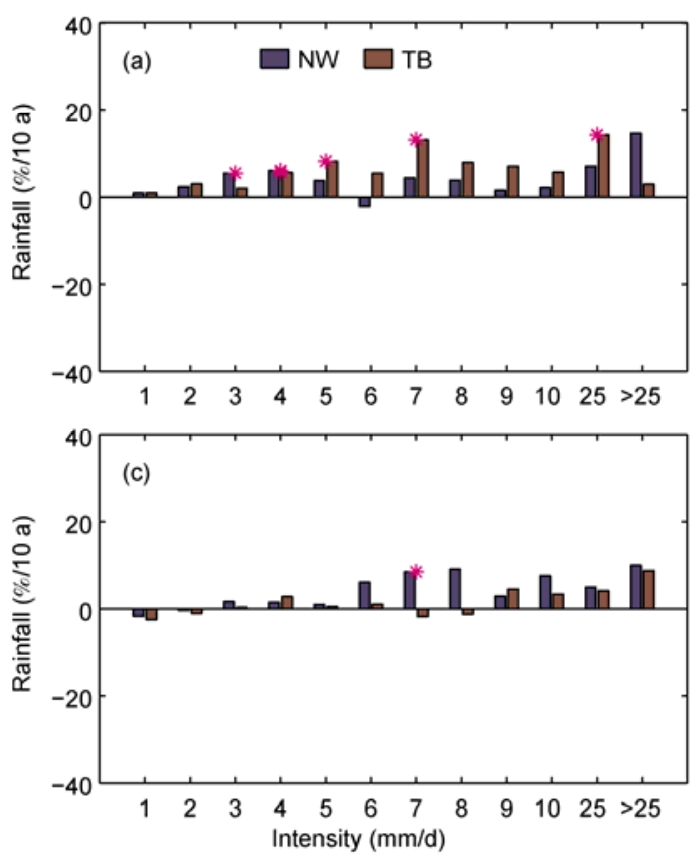

global scale using the GPCP pentad data during the period from 1979 to 2007. Figure 7(a) shows the trend estimates for the whole precipitation spectra using the linear regression method. Solid bars denote that the trends are significant (exceeding the $95 \%$ confidence level) on the basis of the $t$-test. It can be clearly observed that extremely light precipitation with daily precipitation less than $2 \mathrm{~mm} / \mathrm{d}$ and heavy precipitation of more than $13 \mathrm{~mm} / \mathrm{d}$ show increasing trends. Furthermore, the amplification is positively related to the precipitation intensity. Heavy precipitation tends to have a larger increase. By contrast, small precipitation between $2-13 \mathrm{~mm} / \mathrm{d}$ has declined. Overall, the magnitude of precipitation increase is greater than that of the precipitation decrease, implying an increase of mean precipitation intensity.

Global warming has been widely recognized to be a favorable factor for precipitation resulting from increased water vapor in the atmosphere [1,3]. Based on the ClausiusClapeyron equation, the saturation water-vapor pressure rises with temperature at approximately $7 \% / \mathrm{K}$ [1], which implies an increase of precipitable water at the same rate regarding the marginal change in relative humidity predicted in global climate models [33]. Figure 7(b) compares the precipitation amount for each category in the two coldest years (1984 and 1985) and two warmest years (1998 and $2005)$ averaged over $60^{\circ} \mathrm{S}-60^{\circ} \mathrm{N}$. The pattern of differences between warm and cold years is consistent with the trend of precipitation spectra from 1979 to 2007, with extremely light and heavy precipitation in warmer years being greater than that in colder years. Considering that the global mean surface temperature has increased significantly by $0.14^{\circ} \mathrm{C} /$ decade during this period, this uniform pattern verifies the
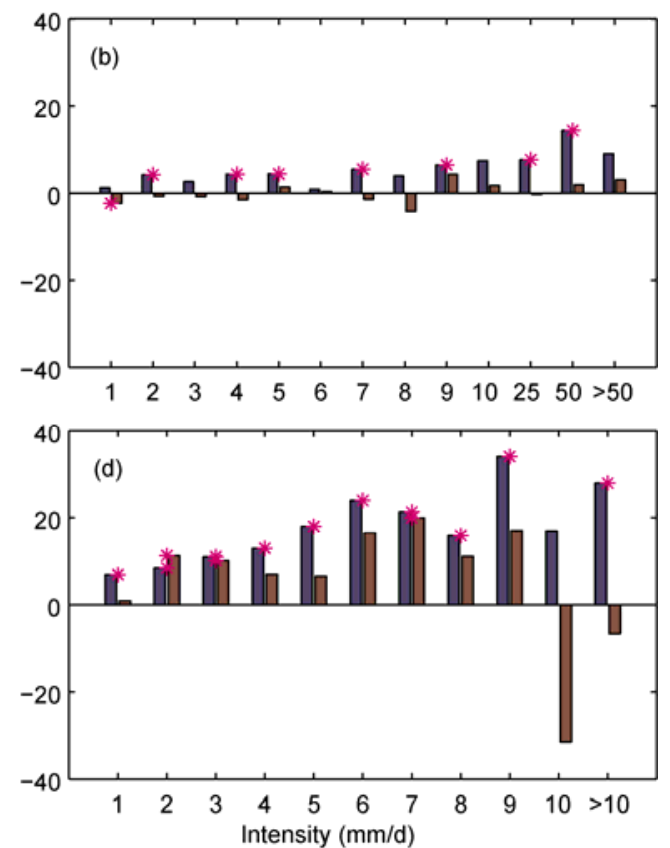

Figure 6 Same as Figure 4, except for west China (Area III), including northwest China and Tibetan area. 

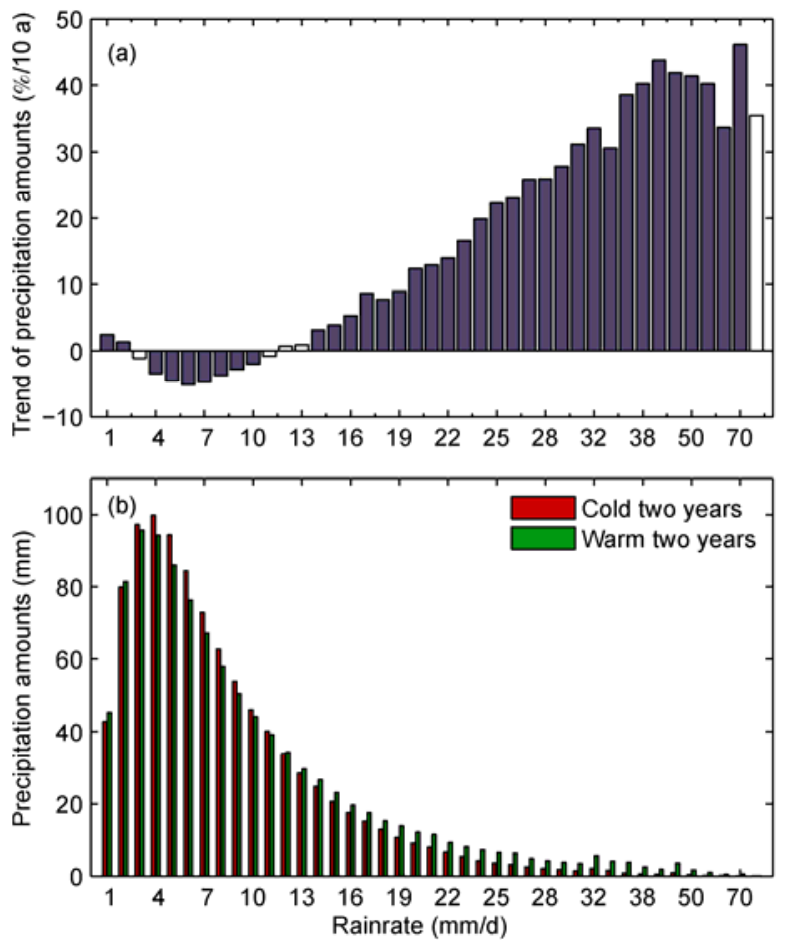

Figure 7 (a) Trend of precipitation spectra during 1979-2007; (b) precipitation spectra comparisons between two warmer years (1998 and 2005) and two colder years (1984 and 1985) over $60^{\circ} \mathrm{S}-60^{\circ} \mathrm{N}$.

substantial implication of global warming on precipitation characteristics.

\subsection{Responses of global precipitation spectra to global warming}

To quantify the response of precipitation intensity to global temperature increase, we adopt the new approach introduced by Liu et al. [20]. They analyzed the relationship between extreme precipitation and global temperature using the differences of precipitation between every two years instead of raw time series of precipitation, as in most studies. In such a way, contributions of global temperatures to changes in precipitation can be extracted effectively. Their study showed that the difference in extreme precipitation tended to be quasi-linearly proportional to the difference in global temperature. Taking advantage of their new finding, we calculate the quasi-constant changing rate for each bin, which is expressed as the change of precipitation amount divided by the maximum change in global temperature of $0.48^{\circ} \mathrm{C}$ during the studied period.

Figure 8 presents the percentage change of precipitation amount in response to a one-degree increase of global temperature for each bin over $60^{\circ} \mathrm{S}-60^{\circ} \mathrm{N}$. The rate of amplification of extreme precipitation to atmospheric warming is marked in the figure. It can be observed that most of the precipitation ranging from light to medium (1.68-12.6 $\mathrm{mm} / \mathrm{d}$ ) has decreased uniformly, with a maximum decline of $27.1 \% /{ }^{\circ} \mathrm{C}$, in Bin $5(4.94-6.23 \mathrm{~mm} / \mathrm{d})$. By contrast, a sub-

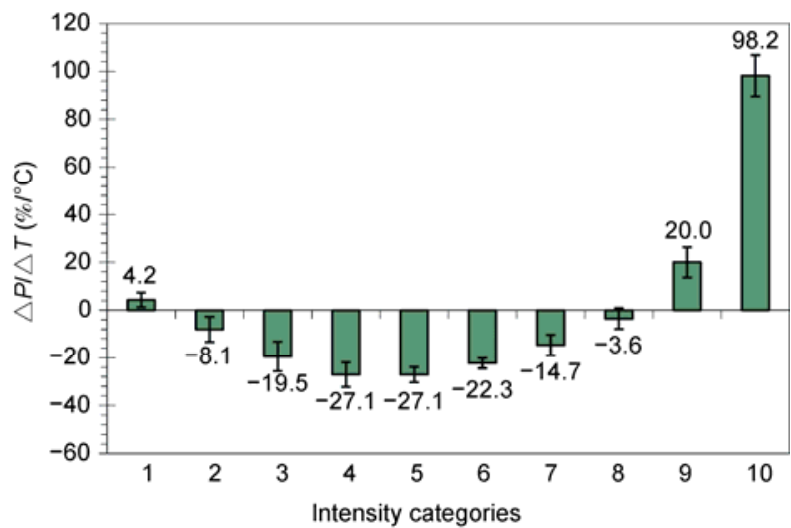

Figure 8 Quasi-constant changing rate of 10 bins of precipitation intensity for each degree increase in global temperature over $60^{\circ} \mathrm{S}-60^{\circ} \mathrm{N}$. The vertical error bar denotes one standard deviation.

stantial increase accompanied with global warming is observed for extremely heavy precipitation with daily precipitation greater than $6.23 \mathrm{~mm} / \mathrm{d}$ (Bin 9 and Bin 10) as well as very light precipitation of less than $1.68 \mathrm{~mm}$ (Bin 1). The magnitude of precipitation increase for the heaviest precipitation, with daily precipitation more than $17.6 \mathrm{~mm} / \mathrm{d}$ (Bin 10 ), is estimated to be $98.2 \% /{ }^{\circ} \mathrm{C}\left(8.6 \% /{ }^{\circ} \mathrm{C}\right)$, and value in the bracket is one standard deviation.

We further calculate the quasi-constant changing rate over $20^{\circ}-40^{\circ} \mathrm{N}$ with increasing global mean surface temperature and compare the similarity and difference over land and ocean areas. As shown in Figure 9, the changing patterns of the whole precipitation spectra with increased temperature over land are similar to those over the ocean despite the different variations in magnitude. Quantitatively, both the reductions of light and medium precipitation and amplification of heavy precipitation over land are smaller than those over the ocean, which may be related to the limited water-vapor supply over land. Unlike the ocean, there is no abundant moist source over land. Limited water supply will suppress the increase of extremely heavy precipitation to some degree. Another obvious difference is the change of very little precipitation (Bin 1). For this category, precipita-

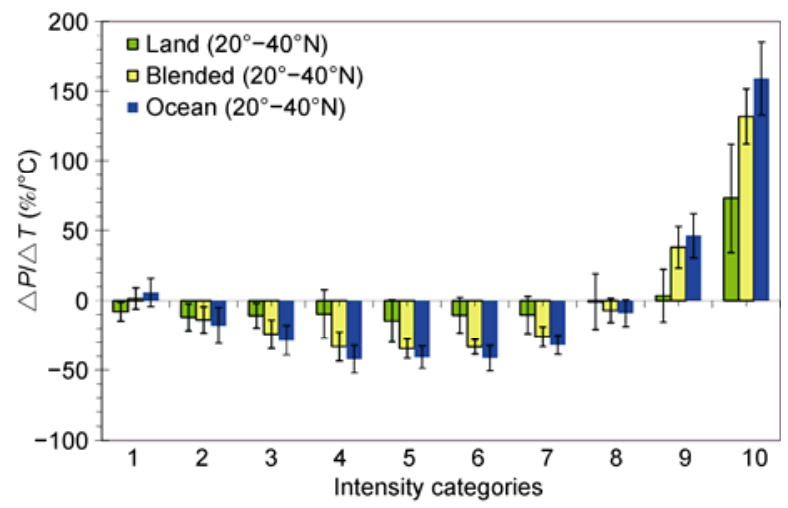

Figure 9 Same as in Figure 8, except for the land, ocean and blended areas over $20^{\circ}-40^{\circ} \mathrm{N}$. 
tion has increased over the ocean as global temperature rises. However, this type of precipitation over land shows an opposite declining tendency $\left(-7.9 \% /{ }^{\circ} \mathrm{C}\right)$, which may be connected with different precipitation characteristics over land and ocean. The precipitation over ocean can be rather intense during a robust convection process. Plentiful water vapor within hours may be graded into slight precipitation through an average over consecutive 5 days. Over land, the decrease of light precipitation may be connected with human activities, ranging from increased anthropogenic aerosols from air pollution to changed land-surface coverage. In the past, many studies attributed the decreased light precipitation to increased aerosols $[6,8]$. These studies argued that increased aerosols can significantly increase the cloud droplet number concentration and reduce droplet sizes, leading to a significant decline in raindrop concentration. Delayed precipitation is beneficial for water-vapor accumulation in the atmosphere, and it rains until the air becomes saturated. This process actually decreases the light precipitation and ultimately turns it into heavy precipitation.

\subsection{Responses of regional precipitation spectra to global warming}

Following the same method, we also calculate the quasiconstant changing rate of the 10 bins of precipitation over the eight subregions in China from 1961 to 2009. The results are shown in Figure 10. Despite the large uncertainty, a uniform variation pattern can be observed in which light precipitation demonstrates a negative tendency, while heavy precipitation demonstrates a positive trend as global temperature rises. Furthermore, the smaller the precipitation intensity, the more the precipitation decreases, and vice versa. This pattern implies a shift from light to heavy precipitation under a warming scenario.

A comparison with results obtained from the GPCP pentad data shows that the whole pattern of precipitation change in China is consistent with the latter segment, from Bin 5 to Bin 10, of the global spectra. In view of the different temporal resolutions, we suspect that the average calculation process of the GPCP pentad data has produced an artificial result for so-called light precipitation (from approximately Bin 1 to Bin 4). Further study of precipitation with high temporal resolution is needed to test our hypothesis. Considering this possible factor, the precipitation changes of different intensities over global and regional scales are qualitatively consistent, with a shift of the precipitation spectra from light to heavy precipitation. The main discrepancy rests on the extent of uncertainty. Over land area, there may be other parameters affecting the change of precipitation besides the temperature.

In fact, regional precipitation is more complicated. As mentioned earlier, non-homogeneous land surface coverage and topography can change energy, momentum and water fluxes locally as well as the water vapor transport among regions. In addition, increased aerosols from human activities may change precipitation characteristics through cloud microphysical processes more locally and regionally. As a result, it is more difficult to extract the relative contributions of different factors.

\section{Conclusions and discussions}

In this study, we examined the trend of the whole precipitation intensity spectra in China during the period from 1961

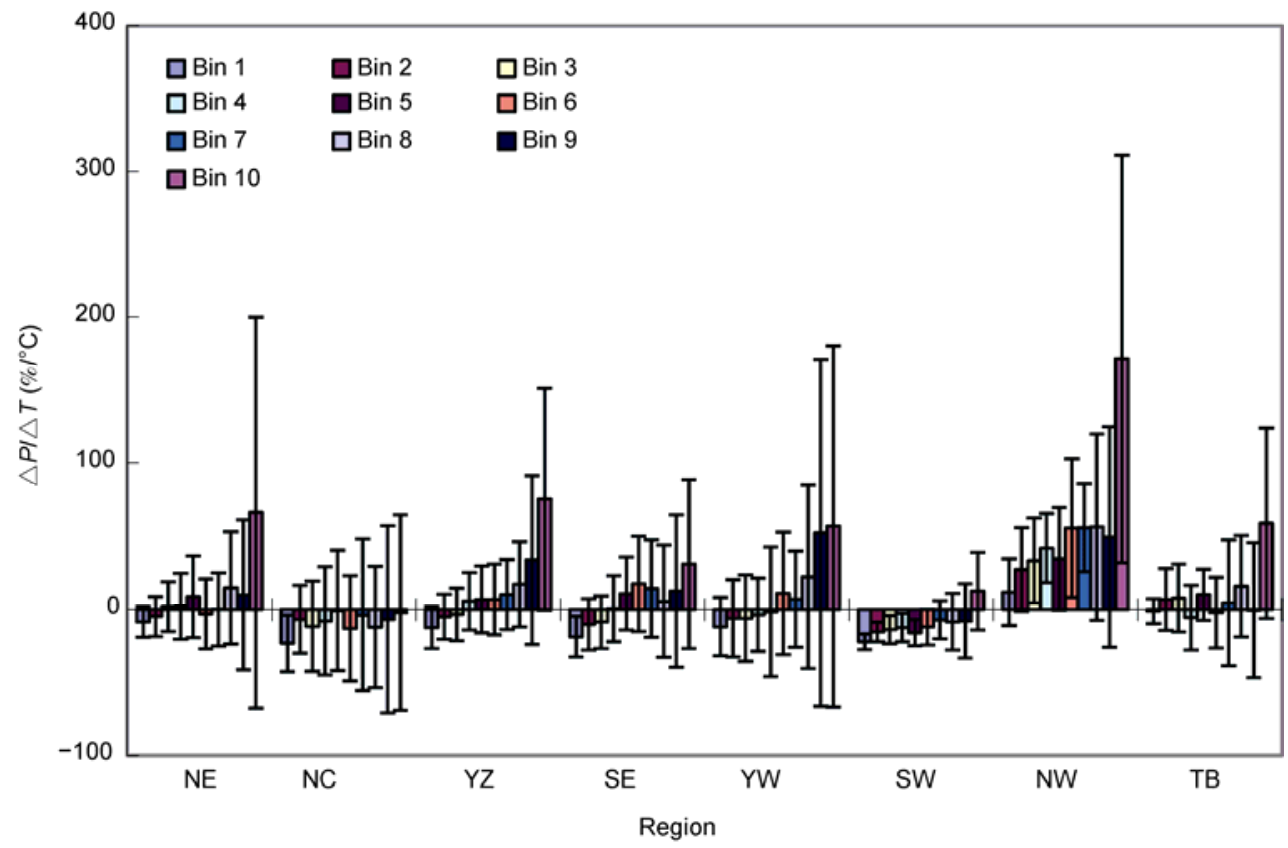

Figure 10 Same as in Figure 8, except for eight subregions in China. 
to 2008 using daily observational data from 477 surface stations provided by the NMIC/CMA. In addition, the precipitation records of 10 intensity bins on the global scale were studied using the GPCP pentad precipitation data from 1979 to 2007. Coupled analysis with global temperature was carried out following a relatively new technique introduced by Liu et al. [20]. The following conclusions are drawn through the above analysis:

(1) The observational data revealed a spatially coherent decreasing trend of trace precipitation over China from 1961 to 2008. In contrast, the pattern of detectable light precipitation $(<1.0 \mathrm{~mm})$ shows a negative trend over east China but a positive trend over West China, which implies that the causes of trace and detectable light precipitation may be somewhat different. Because trace precipitation is extremely fine and generally smaller than $0.5 \mathrm{~mm}$ in diameter, it is easily evaporated before reaching the ground. Compared with relatively larger droplets of light precipitation, trace precipitation is more sensitive to increased temperature. Rising temperature can substantially restrain the frequency of trace precipitation.

(2) For detectable precipitation, the trend showed typical regional and seasonal characteristics. Precipitation has declined consistently in the summer and autumn seasons over the northern part of East China, while a uniform increase in this area has been observed for the entire precipitation spectra in winter. Over the southern part of East China, a significant positive trend in heavy precipitation and a negative trend in light and medium precipitation were both observed in summer and winter. Meanwhile, a coherent decline for the entire precipitation spectra in spring and autumn was detected. In the western China, the precipitation shows a consistent increase despite the decreased trace precipitation events.

(3) Composite analysis demonstrates an intimately positive correlation between precipitation intensity and global temperature. Accompanied by ever-increasing temperature, a substantial decline in medium precipitation and an increase in extremely heavy and light precipitation were observed over $60^{\circ} \mathrm{S}-60^{\circ} \mathrm{N}$ globally, but $20^{\circ}-40^{\circ} \mathrm{N}$ over the ocean only.

(4) Comparisons of the response of precipitation spectra to global warming between land and ocean areas over $20^{\circ}-40^{\circ} \mathrm{N}$ show significant discrepancies for the change of light precipitation (Bin 1) apart from different magnitudes for other categories. The estimated quasi-constant changing rate for the bottom $10 \%$ bin over the ocean is a $5.8 \% / \mathrm{K}$ increase, while that over land has an opposite trend, a decrease by $7.9 \% / \mathrm{K}$. This difference may be partly due to more aerosol particles over land that tend to suppress light precipitation [8]. With respect to the smaller magnitudes of other categories over land than those over the ocean, we hypothesize that this is associated with limited available water vapor over land, which is affected by local evapotranspiration, especially the transportation from the ocean by atmospheric circulation. As for the precipitation spectra change responding to global warming in China and its eight subregions, similar patterns are detected except for relatively larger uncertainties. This result confirms our hypothesis that there may be other factors affecting the precipitation spectra distribution on a regional scale.

The present study focused on the trend of the entire precipitation spectra and compared the implications of rising temperature on precipitation over different spatial scales with the goal of finding other possible causes that affect the precipitation spectra over land on a local scale. Our results have shown that rising temperature can strengthen precipitation intensity with a shift from light to heavy precipitation. Further research is needed to investigate the potential impacts of aerosols on precipitation spectra, among other things.

This work was supported by the National Basic Research Program of China (2011CB952002), the National Natural Science Foundation of China (40810059003) and the project funded by the Priority Academy Program Development of Jiangsu Higher Education Institutions. We thank two anonymous reviewers for their suggestions on improving the manuscript.

1 Trenberth K E, Dai A G, Rasmussen R M, et al. The changing character of precipitation. Bull Amer Meteorol Soc, 2003, 84: 12051217

2 Fowler A M, Hennessy K J. Potential impacts of global warming on the frequency and magnitude of heavy precipitation. Nat Hazards, 1995, 11: 283-303

3 Trenberth K E. Atmospheric moisture residence times and cycling: Implications for rainfall rates and climate change. Clim Change, 1998, 39: 667-694

4 Goswami B N, Venugopal V, Sengupta D, et al. Increasing trend of extreme rain events over India in a warming environment. Science, 2006, 314: 1442-1444

5 Ramanathan V, Crutzen P J, Kiehl J T, et al. Aerosols, climate, and the hydrological cycle. Science, 2001, 294: 2119-2124

6 Rosenfeld D, Lohmann U, Raga G, et al. Flood or drought: How do aerosols affect precipitation? Science, 2008, 321: 1309-1313

7 Gong D, Ho C, Chen D, et al. Weekly cycle of aerosol-meteorology interaction over China. J Geophys Res, 2007, 112: D22202

8 Qian Y, Gong D, Fan J, et al. Heavy pollution suppresses light rain in China: Observations and modeling. J Geophys Res, 2009, 114: D00K02

9 Lau K M, Wu H T. Detecting trends in tropical rainfall characteristics, 1979-2003. Int J Climatol, 2007, 27: 979-988

10 Fujibe F, Yamazaki N, Katsuyama M, et al. The increasing trend of intense precipitation in Japan based on four-hourly data for a hundred years. SOLA, 2005, 1: 41-44

11 Gong D Y, Wang S W. Severe summer rainfall in China associated with enhanced global warming. Climate Res, 2000, 16: 51-59

12 Soden B J, Wetherald R T, Stenchikov G L, et al. Global cooling after the eruption of Mount Pinatubo: A test of climate feedback by water vapor. Science, 2002, 296: 727-730

13 Sun Y, Solomon S, Dai A, et al. How often will it rain? J Clim, 2007, 20: 4801-4818

14 Lenderink G, Van Meijganrd E. Increase in hourly precipitation extremes beyond expectations from temperature changes. Nat Geosci, 2008, 1: 511-514

15 Easterling D R, Meehl G A, Parmesan C, et al. Climate extremes: Observations, modeling, and impacts. Science, 2000, 289: 2068-2074

16 Alpert P, Ben G T, Bahrahad A, et al. The paradoxical increase of Mediterranean extreme daily rainfall in spite of decrease in total values. Geophys Res Lett, 2002, 29: 1536 
17 Qian W, Fu J L, Yan Z W. Decrease of light rain events in summer associated with a warming environment in China during 1961-2005. Geophys Res Lett, 2007, 34: L11705

18 Liu B H, Xu M, Henderson M. Where have all the showers gone? Regional declines in light precipitation events in China, 1960-2000. Int J Climatol, 2010, 31: 1177-1191

19 Fu J L, Qian W, Lin X, et al. Trends in graded precipitation in China from 1961 to 2000. Adv Atmos Sci, 2008, 25: 267-278

20 Liu S C, Fu C B, Shiu C J, et al. Temperature dependence of global precipitation extremes. Geophys Res Lett, 2009, 36: L17702

21 Ye B, Yang D, Ding Y, et al. A bias-corrected precipitation climatology for China. J Hydrometeorol, 2004, 5: 1147-1160

22 Xie P, Janowiak J E, Arkin P A, et al. GPCP pentad precipitation analyses: An experimental dataset based on gauge observations and satellite estimates. J Clim, 2003, 16: 2197-2214

23 Smith T M, Reynolds R W, Peterson T C, et al. Improvements to NOAA's historical merged land-ocean surface temperature analysis (1880-2006). J Climate, 2008, 21: 2283-2296

24 Yan Z W, Yang C. Geographic Patterns of extreme climate changes in China during 1951-1997 (in Chinese). Clim Environ Res, 2000, 5: 267-272

25 Liu B, Xu M, Henderson M, et al. Observed trends of precipitation amount, frequency, and intensity in China, 1960-2000. J Geophys
Res, 2005, 110: DO8103

26 Wang Y, Yan Z W. Trends in seasonal precipitation over China during 1961-2007. Atmos Oceanic Sci Lett, 2009, 2: 165-171

27 Wang H J. The weakening of the Asian monsoon circulation after the end of 1970s. Adv Atmos Sci, 2001, 18: 376-386

28 Li J, Dong W J, Yan Z W. Changes of climate extremes of temperature and precipitation in summer in eastern China associated with changes in atmospheric circulation in East Asia during 19602008. Chin Sci Bull, 2012, 57: 1856-1861

29 Shi Y F, Shen Y P, Li D L, et al. Discussion on the present climate change from warm-dry to warm-wet in northwest China (in Chinese). Quat Sci, 2003, 23: 152-164

30 Zhai P, Ren F M, Zhang Q. Detection of trends in China's precipitation extremes. Acta Meteorol Sin, 1999, 57: 208-216

31 Li D L, Wei L, Cai Y, et al. The present facts and the Future Tendency of the climate change in Northwest China (in Chinese). J Glaciol Geocryol, 2003, 25: 135-142

32 Yang X D, Zhai P M. Changes in precipitation intensity, frequency, and total in Northwest China (in Chinese). Sci Technol Rev, 2005, 23: 24-26

33 Bony S, Colman R, Kattsov V M, et al. How well do we understand and evaluate climate change feedback processes? J Clim, 2006, 19: 3445-3482

Open Access This article is distributed under the terms of the Creative Commons Attribution License which permits any use, distribution, and reproduction in any medium, provided the original author(s) and source are credited. 